Fetal Diagnosis and Therapy
Fetal Diagn Ther 2012;32:295-298

DOI: $\underline{10.1159 / 000341569}$
Received: June 17, 2012

Accepted after revision: June 26, 2012

Published online: October 24, 2012

\title{
Encephalocystocele - Uncommon Diagnosis in Prenatal Medicine
}

\author{
Robert Dankovcik $^{b, c}$ Vlasta Vyhnalkova ${ }^{d}$ Stela Muranska ${ }^{c}$ Eduard Kucera $^{f}$ \\ Maria Korpova ${ }^{\mathrm{e}}$ Anna Plichtova ${ }^{\mathrm{a}, \mathrm{e}}$ Maria Miklosova ${ }^{\mathrm{e}}$ Vladimir Ferianec $^{\mathrm{a}}$ \\ Jan Evangelista Jirasek ${ }^{g}$ Marek Dudas $^{\mathrm{e}}$ \\ aSecond Department of Obstetrics and Gynecology, Comenius University and University Hospital, Bratislava, \\ bSecond Department of Obstetrics and Gynecology, P. J. Safarik University and L. Pasteur University Hospital, \\ ${ }^{c}$ Center for Prenatal Diagnosis, s.r.o., ${ }^{d}$ Department of Forensic Medicine and Pathology, Health Care Surveillance \\ Authority of Slovak Republic, and ' $F$ Fetal Medicine Program, Department of Cell Biology, P. J. Safarik University, \\ Kosice, Slovakia; fDepartment of Gynecology and Obstetrics, Third Faculty of Medicine, Charles University in \\ Prague, and 9Institute for Care of Mother and Child, Prague, Czech Republic
}

\section{Key Words}

Cephalocele $\cdot$ Encephalocystocele $\cdot$ Meningocele

\begin{abstract}
Encephalocystocele is a developmental malformation characterized by brain herniation accompanied with extracranial cystic protrusion of the ventricular system. This nosological unit is often overlooked and insufficiently classified merely as encephalocele. Herein, two exceptionally clear cases of the parieto-occipital cranioschisis with encephalocystocele and congenital hydrocephalus of the lateral ventricles are documented with 2-dimensional/3-dimensional sonographic images and the corresponding MRI findings. In both cases, prenatal diagnosis was confirmed by autopsy.
\end{abstract}

Copyright $\odot 2012$ S. Karger AG, Basel
(C) 2012 S. Karger AG, Basel

$1015-3837 / 12 / 0324-0295 \$ 38.00 / 0$

Fax +4161306 1234

E-Mail karger@karger.ch

www.karger.com
Accessible online at: www.karger.com/fdt

\section{Clinical Case 1}

Unilateral Encephalocystocele in an Otherwise Normal Brain

A single live fetus [gestation week (GW) 18; healthy mother/ age 29, gravida 3, para 2] affected with prominent dilatation of the left lateral ventricle and atrophic brain tissue was identified by sonography (fig. 1a, b). MRI in GW 24 revealed parieto-occipital encephalocystocele (fig. 1c, d). Cesarean section at GW 38 delivered a full-term newborn girl, eutrophic, $4,020 \mathrm{~g}, 51 \mathrm{~cm}$, APGAR score 7/6, normal motorics, head circumference $44 \mathrm{~cm}$. No malformations of the heart, kidney or other organs were found on sonography; small amounts of milk were ingested per os. The soft parieto-occipital protrusion (fig. le, f) showed no cerebrospinal fluid leakage or bleeding. Dyspnea and cyanosis developed $3.5 \mathrm{~h}$ postpartum; oxygen inhalation restored blood saturation to $99 \%$, but inoperability was stated by a neurosurgeon. Sudden apnea and bradycardia appeared $5.5 \mathrm{~h}$ later, followed by asystolia after another hour. Autopsy revealed a largely normal brain with a normal left frontal lobe and a normal anterior portion of the left lateral ventricle. The expanded posterior part of the left lateral ventricle was covered in thinned differentiated brain tissue and herniated via the distended fontanella occipitalis (fig. 1g, h). 
Fig. 1. Case 1: unilateral encephalocystocele $\left(^{*}\right)$ in the otherwise normal brain. For easy visual comparison, all head and brain images are oriented with the front facing the right side, right brain hemisphere up (all except c, e) or above the image plane/ facing the observer (c, e). a Two-dimensional ultrasound at GW 18, horizontal section through the fetal head with normal right lateral ventricle, dilatation of the left lateral ventricle, and left occipital encephalocystocele $\left(^{*}\right)$. b Three-dimensional rendering at the same age and on the same sonographic plane as in a. c Sagittal fast-spin MRI at GW 24 shows a parieto-occipital skull defect (widened and perforated fontanella occipitalis) with encephalocystocele (asterisk in cystic protrusion of the left ventricle) covered in an additional protruding sac (meningocele, mc) formed by the dura mater (labeled arrowhead). d Horizontal fast T2-weighted MRI at GW 24 reconfirms the herniated brain and meninges together with a posterior part of dilated left lateral ventricle $\left(^{*}\right)$. e Dura-covered protruding parieto-occipital encephalocystocele $\left({ }^{*}\right) 1 \mathrm{~h}$ after live delivery. f Horizontal two-dimensional sonogram $6 \mathrm{~h}$ after the delivery shows persisting extreme dilatation of the ventricular system, with thinned brain tissue on the left side forming a 1-cm-thick layer (arrows). g, h Brain autopsy, basal (inferior) view. Normal overall anatomy and brain thickness was revealed, except for the herniated $\left(^{*}\right)$ posterior portion of the left lateral ventricle, which was dilated and surrounded by thinned and smoothened brain tissue (lissencephaly) of the temporal and occipital lobe . c.inf. $=$ Cornu inferius ventriculi lat; c.post. = cornu posterius ventriculi lat; crbl./flip = cerebellum, flipped frontally; $\mathrm{F}=$ frontal direction; $\mathrm{L}=$ left side; lob. occipit. = lobus occipitalis cerebri; lob. temp. = lobus temporalis cerebri; $\mathrm{LV}=$ left lateral ventricle; $\mathrm{mc}=$ meningocele; $\mathrm{R}=$ right side; $\mathrm{RV}=$ right lateral ventricle; sep. pell. $=$ septum pellucidum; thal.sin.$=$ left thalamus.
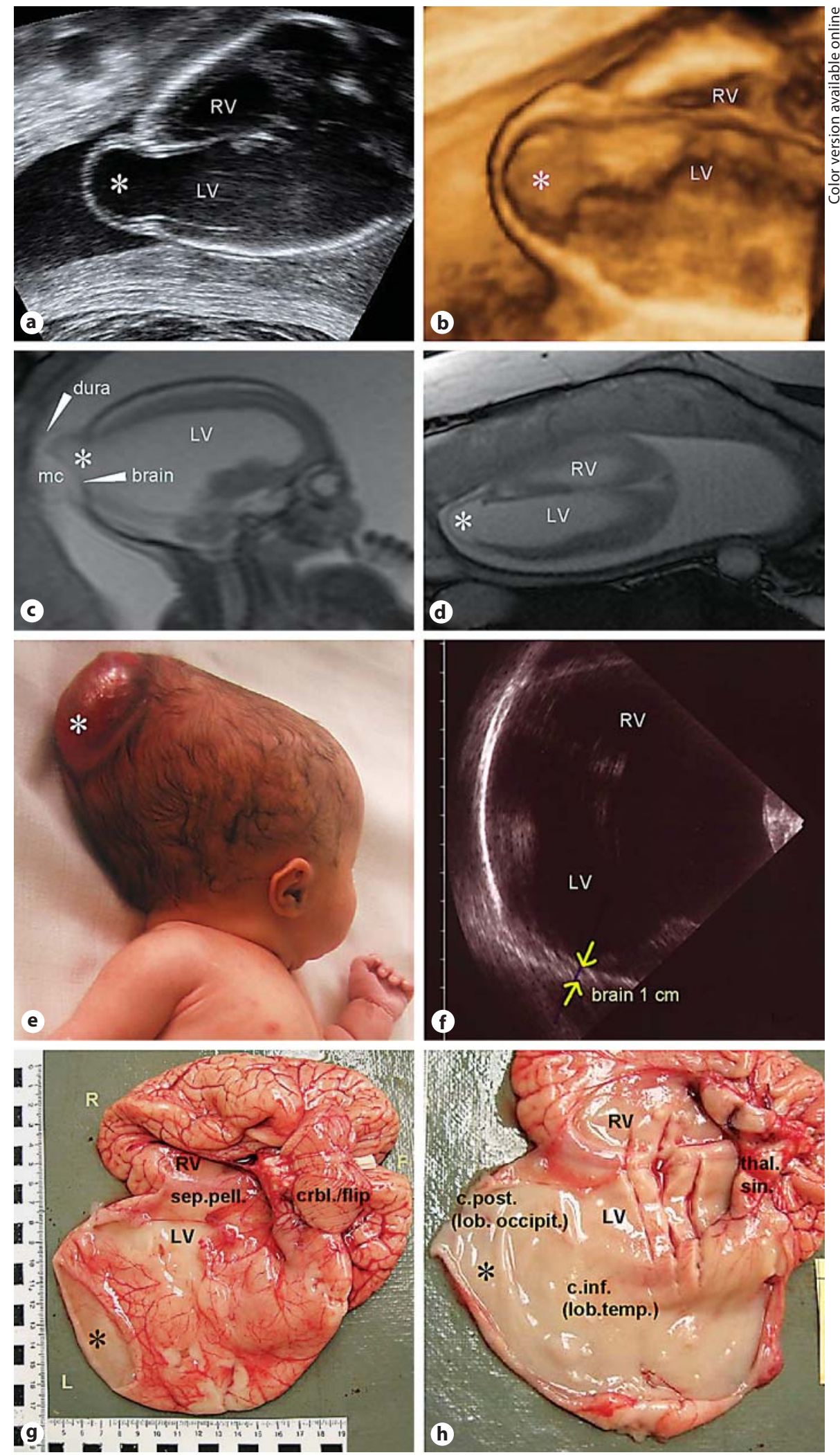

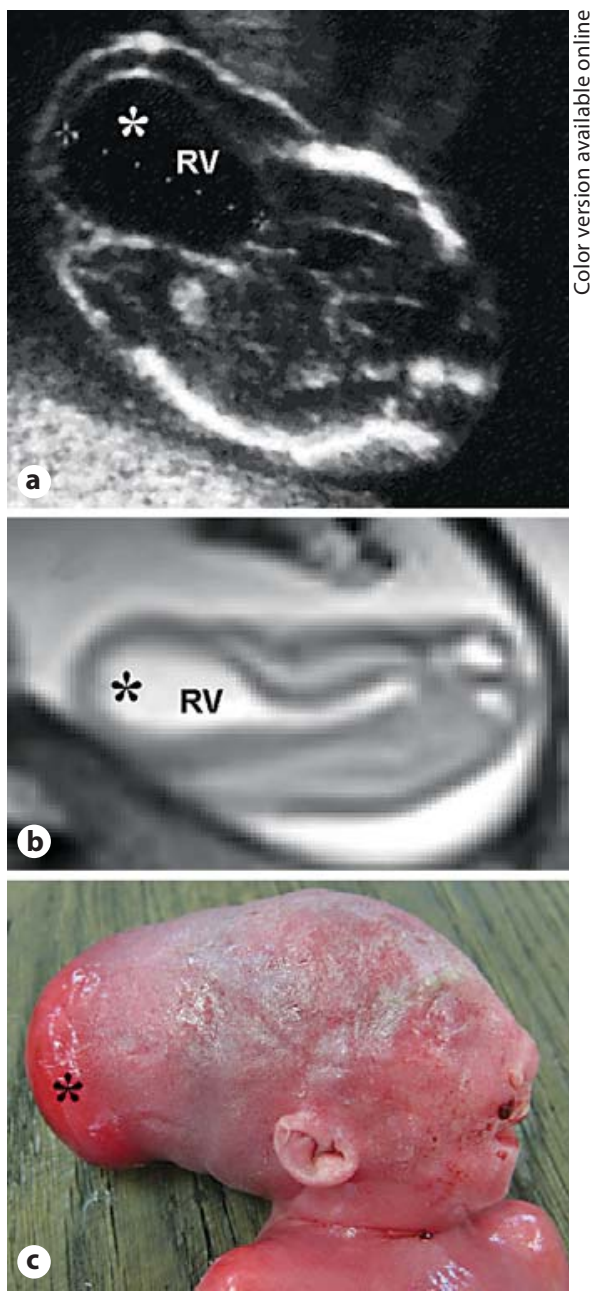

Fig. 2. Case 2: Unilateral encephalocystocele $\left(^{*}\right)$ in a fetus with multiple brain anomalies. For easy visual comparison, all head and brain images are oriented with the front facing the right side, right brain hemisphere up (a, b) or facing the camera (c). a Twodimensional ultrasound, horizontal section of the fetal head at GW 20 with dilatation of the right lateral ventricle protruding together with a thinned brain through a parieto-occipital skull defect. b Sagittal fast-spin T2-weighted MRI at GW 21 shows asymmetric dilatation of the posterior horn of the right lateral ventricle. c Aborted fetus, GW 22. Head with the skin defect covering the bulging parieto-occipital encephalocystocele $\left(^{*}\right)$. RV = Right lateral ventricle.

\section{Clinical Case 2}

Unilateral Encephalocystocele Associated with Severe Brain Malformations

Right parieto-occipital encephalocystocele (fig. 2a) was identified by sonography in a single live fetus (GW 20; healthy mother/age 29, gravida 1, para 0). MRI at GW 21 reconfirmed unilateral hemisphere dilatation, with the wall of widely communicat- ing herniation sac formed by thin atrophic cerebral tissue (fig. 2b). The frontal, parietal and temporal brain lobes were bilaterally adequately developed. Pathologies included agenesis of the corpus callosum, absence of the fourth ventricle and cisterna magna, hypoplastic cerebellar hemispheres and cerebellar tonsils protruding into the foramen magnum (suggesting Chiari malformation I-II). The spinal cord, thorax and abdominal organs were normal. Biochemical screening for neural tube defects and Down syndrome at GW 16 were negative and karyotype was normal (46, XY). Postabortum examination at GW 22 (fig. 2c) revealed no other anomalies and no cerebrospinal fluid leakage or bleeding.

\section{Discussion}

Cephalocele is a general term referring to anomalies characterized by external herniation of the skull contents, while the more detailed terms meningocele and encephalocele refer to prolapsing meninges or brain, respectively. Encephalocystocele is a special, rarely mentioned nosological subcategory, where the cerebral ventricular system also participates in protrusion beyond the skull outline (in our 2 cases, the herniation occurred via the disrupted fontanella occipitalis, seu fonticulus minor/f. posterior/f. triangularis being the locus minoris resistentiae). Although the brain and its ventricular system are enlarged and protrude above the skull niveau, generalized hydrocephalus is not necessarily a part of the clinical picture: clinical case 1 clearly demonstrates localized hydrocephalus affecting only the herniating, thinned and smoothened brain lobes.

During routine ultrasonography, encephalocystocele with herniated expanded ventricles and extremely thinned brain may be easily misinterpreted or misrecognized as meningocele (simple meningeal herniation sac). However, precise distinction and a differential diagnosis are extremely important in terms of prognosis: while meningocele per se is a benign and easily correctable anomaly, encephalocystocele is a serious condition with infaust prognosis or else requiring extensive planning in advance and neonatal brain surgery [1].

Further confusion, both nomenclature-related and diagnostic, can emerge when encephalocystocele is combined with meningocele. The eventual cystic nature of this additional meningeal protrusion may contribute to the formation of a bilayered or doubled herniation sac (dubbed 'meningocysto-encephalocystocele'), seen clearly in case 1 (fig. 1c). The same picture also shows that the cerebral (innermost) component of the double herniation bag was reverse-impressed toward the endocranial cavity in the living fetus, while it readily ruptured when touched 
at autopsy (asterisk in fig. 1g). This substantiates a speculation that antenatal displacements, folding or perhaps rupture of extremely thinned brain inside the encephalocystocelic pouch could imitate meningocele, or otherwise mislead the sonographist. In conclusion, encephalocystocele should be routinely suspected and carefully differentiated in all cases of prenatal sonographic diagnosis of any cephalocele.

\section{Acknowledgements}

This work was supported by the projects APVV VVCE-000107, ASFEU ITMS 26220120024-SEPO and the Slovak Ministry of Health grant No. 2007/65-UPJS-02.

Reference

Yamada K, Miura M, Mastumoto J, Uchino T, Kondo Y, Ushio Y: An occipital encephalocystocele involving both sides of the lateral ventricles. Pediatr Neurosurg 2000;33:279282. 